\title{
Upper limits to the SN1006 multi-TeV gamma-ray flux from HESS observations
}

\author{
F. Aharonian ${ }^{1}$, A. G. Akhperjanian ${ }^{2}$, K.-M. Aye ${ }^{3}$, A. R. Bazer-Bachi ${ }^{4}$, M. Beilicke ${ }^{5}$, W. Benbow ${ }^{1}$, D. Berge ${ }^{1}$, \\ P. Berghaus ${ }^{6, \star}$, K. Bernlöhr ${ }^{1,7}$, C. Boisson ${ }^{8}$, O. Bolz ${ }^{1}$, C. Borgmeier ${ }^{7}$, F. Breitling ${ }^{7}$, A. M. Brown ${ }^{3}$, \\ J. Bussons Gordo ${ }^{9}$, P. M. Chadwick ${ }^{3}$, L.-M. Chounet ${ }^{10}$, R. Cornils ${ }^{5}$, L. Costamante ${ }^{1,20}$, B. Degrange ${ }^{10}$,
} A. Djannati-Ataï ${ }^{6}$, L. O’C. Drury ${ }^{11}$, G. Dubus ${ }^{10}$, T. Ergin $^{7}$, P. Espigat $^{6}$, F. Feinstein ${ }^{9}$, P. Fleury ${ }^{10}$, G. Fontaine ${ }^{10}$, S. Funk ${ }^{1}$, Y. A. Gallant ${ }^{9}$, B. Giebels ${ }^{10}$, S. Gillessen ${ }^{1}$, P. Goret ${ }^{12}$, C. Hadjichristidis ${ }^{3}$, M. Hauser ${ }^{13}$, G. Heinzelmann ${ }^{5}$, G. Henri ${ }^{14}$, G. Hermann ${ }^{1}$, J. A. Hinton ${ }^{1}$, W. Hofmann ${ }^{1}$, M. Holleran ${ }^{15}$, D. Horns ${ }^{1}$, O. C. de Jager ${ }^{15}$, I. Jung ${ }^{1,13, \star \star}$, B. Khélifi ${ }^{1}$, Nu. Komin ${ }^{7}$, A. Konopelko ${ }^{1,7}$, I. J. Latham ${ }^{3}$, R. Le Gallou ${ }^{3}$, A. Lemière ${ }^{6}$, M. Lemoine ${ }^{10}$, N. Leroy ${ }^{10}$, T. Lohse ${ }^{7}$, A. Marcowith ${ }^{4}$, C. Masterson ${ }^{1,20}$, T. J. L. McComb ${ }^{3}$, M. de Naurois ${ }^{16}$, S. J. Nolan ${ }^{3}$, A. Noutsos ${ }^{3}$, K. J. Orford ${ }^{3}$, J. L. Osborne ${ }^{3}$, M. Ouchrif ${ }^{16,20}$, M. Panter ${ }^{1}$, G. Pelletier ${ }^{14}$, S. Pita ${ }^{6}$, G. Pühlhofer ${ }^{1,13}$, M. Punch ${ }^{6}$, B. C. Raubenheimer ${ }^{15}$, M. Raue ${ }^{5}$, J. Raux ${ }^{16}$, S. M. Rayner ${ }^{3}$, I. Redondo ${ }^{10,20, \star \star \star}$, A. Reimer $^{17}$, O. Reimer ${ }^{17}$, J. Ripken ${ }^{5}$, L. Rob ${ }^{18}$, L. Rolland ${ }^{16}$, G. P. Rowell ${ }^{1}$, V. Sahakian ${ }^{2}$, L. Saugé ${ }^{14}$, S. Schlenker ${ }^{7}$, R. Schlickeiser ${ }^{17}$, C. Schuster ${ }^{17}$, U. Schwanke ${ }^{7}$, M. Siewert ${ }^{17}$, H. Sol $^{8}$, R. Steenkamp ${ }^{19}$, C. Stegmann ${ }^{7}$, J.-P. Tavernet ${ }^{16}$, C. G. Théoret ${ }^{6}$, M. Tluczykont ${ }^{10,20}$, D. J. van der Walt ${ }^{15}$, G. Vasileiadis ${ }^{9}$, P. Vincent ${ }^{16}$, B. Visser ${ }^{15}$, H. J. Völk ${ }^{1}$, and S. J. Wagner ${ }^{13}$

\begin{abstract}
${ }^{1}$ Max-Planck-Institut für Kernphysik, e-mail: [Conor.Masterson;Gavin.Rowell]@mpi-hd.mpg.de ${ }^{2}$ Yerevan Physics Institute, ${ }^{3}$ University of Durham, ${ }^{4}$ Centre d'Étude Spatiale des Rayonnements, ${ }^{5}$ Universität Hamburg, Institut für Experimentalphysik, ${ }^{6}$ Physique Corpusculaire et Cosmologie, IN2P3/CNRS, Collège de France, ${ }^{7}$ Institut für Physik, Humboldt-Universität zu Berlin, ${ }^{8}$ LUTH, UMR 8102 du CNRS, Observatoire de Paris, ${ }^{9}$ Groupe d'Astroparticules de Montpellier, ${ }^{10}$ Laboratoire Leprince-Ringuet, École Polytechnique ${ }^{11}$ Dublin Institute for Advanced Studies ${ }^{12}$ Service d'Astrophysique, DAPNIA/DSM/CEA, CE Saclay, ${ }^{13}$ Landessternwarte, Königstuhl, ${ }^{14}$ Laboratoire d'Astrophysique de Grenoble, INSU/CNRS, Université Joseph Fourier, ${ }^{15}$ Unit for Space Physics, North-West University, ${ }^{16}$ Laboratoire de Physique Nucléaire et de Hautes Énergies, IN2P3/CNRS, Universités Paris VI \& VII, ${ }^{17}$ Institut für Theoretische Physik, Ruhr-Universität Bochum, ${ }^{18}$ Institute of Particle and Nuclear Physics, Charles University, ${ }^{19}$ University of Namibia, ${ }^{20}$ European Associated Laboratory for Gamma-Ray Astronomy, jointly supported by CNRS and MPG
\end{abstract}

Received 11 December 2004 / Accepted 4 February 2005

\begin{abstract}
Observations of the shell-type supernova remnant SN1006 have been carried out with the HESS system of Cherenkov telescopes during 2003 (18.2 h with two operating telescopes) and 2004 (6.3 h with all four telescopes). No evidence for $\mathrm{TeV} \gamma$-ray emission from any compact or extended region associated with the remnant is seen and resulting upper limits at the $99.9 \%$ confidence level are up to a factor 10 lower than previously-published fluxes from CANGAROO. For SN1006 at its current epoch of evolution we define limits for a number of important global parameters. Upper limits on the $\gamma$-ray luminosity (for $E=0.26$ to $10 \mathrm{TeV}$, distance $d=2 \mathrm{kpc}$ ) of $L_{\gamma}<1.7 \times 10^{33} \mathrm{erg} \mathrm{s}^{-1}$, and the total energy in corresponding accelerated protons, $W_{\mathrm{p}}<1.6 \times 10^{50}$ erg are estimated (for proton energies $E_{\mathrm{p}} \sim 1.5$ to $60 \mathrm{TeV}$ and assuming the lowest value $n=0.05 \mathrm{~cm}^{-3}$ of the ambient target density discussed in literature). Extending this estimate to cover the range of proton energies observed in the cosmic ray spectrum up to the knee (we take here $E_{\mathrm{p}} \sim 1 \mathrm{GeV}$ to $3 \mathrm{PeV}$, assuming a differential particle index -2 ) gives $W_{\mathrm{p}}<6.3 \times 10^{50} \mathrm{erg}$. A lower limit on the post-shock magnetic field of $B>25 \mu \mathrm{G}$ results when considering the synchrotron/inverse-Compton framework for the observed X-ray flux and $\gamma$-ray upper limits.
\end{abstract}

Key words. gamma rays: observations - ISM: supernova remnants

\footnotetext{
* Université Libre de Bruxelles, Faculté des Sciences, Campus de la Plaine, CP230, Boulevard du Triomphe, 1050 Bruxelles, Belgium.

$\star \star$ Now at Washington Univ., Department of Physics, 1 Brookings Dr., CB 1105, St. Louis, MO 63130, USA.

$\star \star \star$ Now at the Department of Physics and Astronomy, Univ. of Sheffield, The Hicks Building, Hounsfield Road, Sheffield S3 7RH, UK.
}

\section{Introduction}

Observations of shell-type supernova remnants (SNR) at multi$\mathrm{GeV}$ to $\mathrm{TeV} \gamma$-ray energies have long been motivated by the idea that they are the prime source of hadronic cosmic-ray (CR) acceleration in our galaxy (see for example Drury et al. 1994 
and Naito \& Takahara 1994). Detection of non-thermal X-ray emission from the young shell-type SNR SN1006 (Koyama et al. 1995; Allen et al. 2001) suggests that SN1006 is a site of electron acceleration to multi-TeV energies. Subsequent arcsecond resolution results from Chandra (Long et al. 2003; Bamba et al. 2003) reveal the presence of several bright nonthermal X-ray arcs concentrated in the NE and SW regions of the SNR, which likely trace the location of strong shocks where electrons are accelerated.

These, and similar X-ray results from a number of other SNR over the past ten years, have provided a priority list of such targets for ground-based $\gamma$-ray telescopes. The results of CANGAROO-I (Tanimori et al. 1998), which suggest TeV $\gamma$-ray emission from the NE rim of SN1006, supported the notion that this SNR is capable of electron acceleration up to energies $\sim 100 \mathrm{TeV}$, when interpreted in the synchrotron/inverse-Compton (IC) framework. The TeV photon spectrum is compatible with a power law of photon index $\Gamma \sim 2.3$ (for $\mathrm{d} N / \mathrm{d} E \sim E^{-\Gamma}$ ) in the energy range 1.5 to $20 \mathrm{TeV}$ (Naito et al. 1999; Tanimori et al. 2001). Later data from the CANGAROO-II 10-m telescope also revealed SN1006 as an emitter of TeV $\gamma$-rays (Hara et al. 2001), yielded a compatible energy spectrum. The stand-alone HEGRA CT1 telescope has also reported a significant excess in observations taken at very large zenith angles and therefore at a high energy threshold $E>18 \mathrm{TeV}$ (Vitale et al. 2003).

Interpretation of these X-ray and ground-based $\gamma$-ray results generally involves the electronic synchrotron/IC and/or hadronic $\pi^{0}$-decay channels. Either channel can dominate, depending on, for example, the density of ambient matter $n$ and magnetic field strength $B$. Detailed sampling of the $\gamma$-ray spectra and morphology are required to disentangle the electronic and hadronic components. The HESS (High Energy Stereoscopic System) experiment, the first of the nextgeneration ground-based $\gamma$-ray detectors to utilise the stereoscopic technique, has observed SN1006 during 2003 and 2004. The prime motivation of these observations, apart from confirming the CANGAROO results, has been to determine the nature of any $100 \mathrm{GeV}$ to $\mathrm{TeV} \gamma$-ray emission from SN1006.

Operating in the Southern Hemisphere, the HESS experiment consists of four identical Cherenkov telescopes, each with mirror area $\sim 107 \mathrm{~m}^{2}$ (Bernlöhr et al. 2003; Cornils et al. 2003; Vincent et al. 2003). All four telescopes have been operating since December 2003. Here, we present results using HESS data taken during 2003 (two telescopes operating in stereo but with considerable dead time of $\sim 50 \%$ due to the lack of a central trigger system (see Funk et al. 2004)). The four-telescope mode of 2004 with the central trigger is the most sensitive configuration for HESS. We now update previous results of Masterson et al. (2003) which described a part of the 2003 dataset analysed without stereoscopic reconstruction (i.e. mono-mode). The large field of view (FoV $\sim 5^{\circ}$ diameter) of HESS easily encompasses the SN1006 shell, and the angular resolution of $\sim 0.1^{\circ}$ attained (event-by-event) permits a search for $\gamma$-ray sources from different regions associated with the SNR.
Table 1. Summary of HESS observations of SN1006.

\begin{tabular}{ccccc}
\hline \hline $\begin{array}{c}\text { Obs. } \\
\text { period }\end{array}$ & Runs & $\begin{array}{c}\text { ON source } \\
\text { livetime }[\mathrm{h}]\end{array}$ & $\langle z\rangle^{a}$ & $\begin{array}{c}\text { Telescopes } \\
\text { in use }\end{array}$ \\
\hline Mar. 2003 & 18 & 4.1 & 23.9 & 2 \\
Apr. 2003 & 21 & 5.0 & 25.4 & 2 \\
May 2003 & 25 & 9.1 & 23.3 & 2 \\
2003 Total & $\mathbf{6 5}$ & $\mathbf{1 8 . 2}$ & $\mathbf{2 4 . 0}$ & \\
\hline May 2004 & 15 & 6.3 & 28.5 & 4 \\
All Total & $\mathbf{8 0}$ & $\mathbf{2 4 . 5}$ & $\mathbf{2 5 . 0}$ & \\
\hline
\end{tabular}

${ }^{a}$ Average zenith angle [deg] of observations.

\section{Analysis and results}

HESS observation runs of $\sim 28$ min duration were taken using the so-called wobble mode in which the tracking position is displaced $\pm 0.5^{\circ}$ in declination with respect to the centre of SN1006 (RA $15^{\mathrm{h}} 02^{\mathrm{m}} 48.4^{\mathrm{s}}$ Dec $-41^{\circ} 54^{\prime} 42^{\prime \prime}$ J2000.0). Runs were accepted for analysis if they met a number of quality control criteria based on the recorded $\mathrm{CR}$ rate, on the number of malfunctioning pixels in each camera and also on the tracking performance. In particular telescope tracking was accurate to $\sim 20$ arcsec for these data and was verified by comparing the locations of bright stars in the camera fields of view. Table 1 summarises observations for those runs meeting quality criteria. Without correction for system deadtime the overall observation times were $30 \mathrm{~h}$ (2003, with $\sim 50 \%$ deadtime) and $7 \mathrm{~h}$ (2004, with $\sim 10 \%$ deadtime). A total of $18.2 \mathrm{~h}$ (2003) and $6.3 \mathrm{~h}$ (2004) livetime of ON source data (where SN1006 is in the FoV) were available for analysis.

Individual telescope events coincident in time were merged for stereo analysis, followed by Cherenkov image reduction. Image reduction employs image "cleaning" that removes camera pixels dominated by skynoise, flat fielding of the camera responses using a LED flasher, and absolute conversion from pixel ADC counts to photoelectron units using conversion constants obtained from special low-illumination runs. Image moments, such as width and length according to Hillas (1985), are used as a basis for rejecting the dominant CR background, is achieved by application of image shape cuts (mean-reduced-scaled-width MRSW and mean-reducedscaled-length $M R S L$ ). A further cut $\theta$, the difference in assumed and reconstructed event directions is also applied. Event directions are reconstructed according to algorithm "1" as detailed by Hofmann et al. (1999). For a point-like source the combination of cuts on shape and direction $\theta_{\text {cut }}<0.14^{\circ}$ allows for rejection of over $99.9 \%$ of CRs whilst retaining $\sim 40 \%$ of $\gamma$-rays above a threshold energy of $260 \mathrm{GeV}$ (2003) and $110 \mathrm{GeV}$ (2004). The full data processing chain and $\mathrm{CR}$ rejection applied to these data were defined a priori using Monte Carlo simulations of $\gamma$-ray and real CR events, and then fully verified on the Crab Nebula. Details can be found in Aharonian et al. (2005).

The skymap in Fig. 1 presents the excess significance for $\gamma$-ray like events (i.e. after cuts) over the RA/Dec plane centred on SN1006. Data from 2003 and 2004 were combined for these 


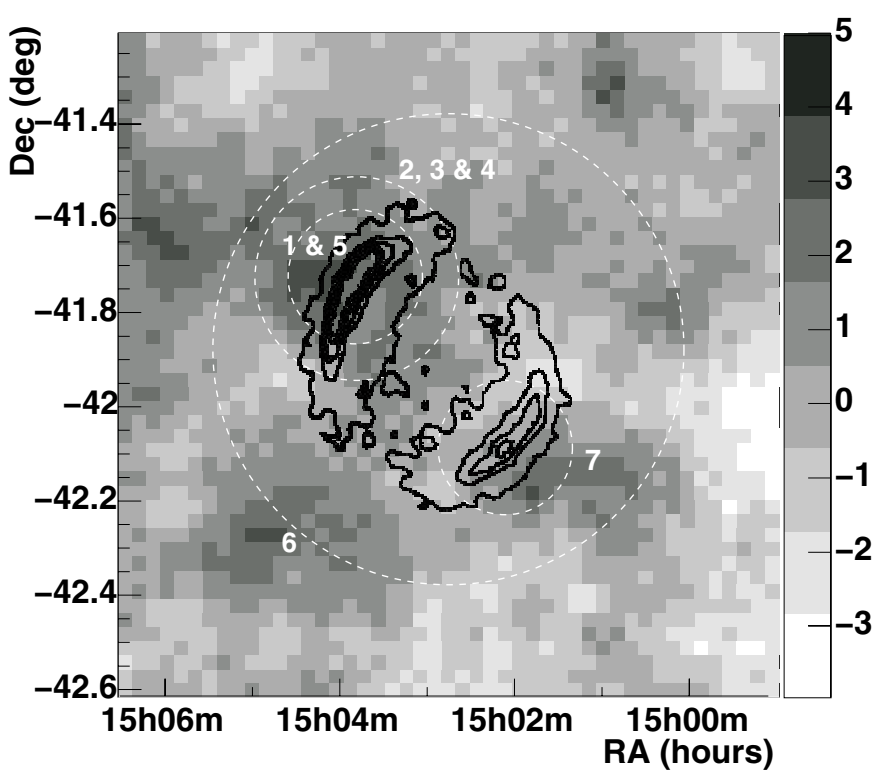

Fig. 1. Skymap of excess significance (according to Eq. (17) of Li \& Ma 1983) centred on SN1006. Indicated are all a priori integration regions used in these analyses (numbered according to the text and Table 2) and ASCA X-ray contours highlighting the X-ray emission from the NE and SW rims. The distribution of significances is fit by a Gaussian distribution with a mean $\mu=-0.016 \pm 0.004$ and sigma $\sigma=1.035 \pm 0.003$.

analyses. At each bin position, $\gamma$-ray-like events are summed within a circle of radius $\theta_{\text {cut }}<0.14^{\circ}$ and the CR background is estimated from a ring region of radius $0.5^{\circ}$ surrounding the source region. The ring region is chosen to give a solid angle ratio $\alpha$ of 1:7 between the source and background regions. The skymap bins of Fig. 1 are therefore correlated. The cut $\theta_{\text {cut }}<0.14^{\circ}$ is appropriate for point-like $\gamma$-ray and marginally extended ( $\sim$ few arcmin) emission according to the HESS point spread function (PSF). No significant excess suggesting $\gamma$-ray emission is evident in the skymap. A very similar skymap is obtained when using the alternative template model (Rowell 2003) as a CR background estimate. Event statistics and $\gamma$-ray flux upper limits at the $99.9 \%$ confidence level, summarised in Table 2, have been calculated for a number of a priori chosen locations based on the X-ray morphology as imaged by ASCA and the results of CANGAROO and HEGRA CT1. The upper limits in the table have assumed a differential photon index of $\Gamma=2$, and later (in Fig. 2) we give the upper limit band for a range of assumed indices (2 to 3 ). The a priori locations, also indicated in Fig. 1, are defined as:

1. HESS Point: upper limit on point-like emission $(E>$ $0.26 \mathrm{TeV}$ ) at the CANGAROO position using $\theta_{\text {cut }}<0.14^{\circ}$ (appropriate for the HESS PSF). This is also used as an upper limit for the NE Rim position.

2. CANGAROO Point: upper limit at the CANGAROO position using a $\theta_{\text {cut }}<0.25^{\circ}$ cut appropriate for the CANGAROO $3.8 \mathrm{~m}$ PSF and selecting events above their energy threshold $(E>1.7 \mathrm{TeV})$.

3. CANGAROO Point 2003: as above using only 2003 data.
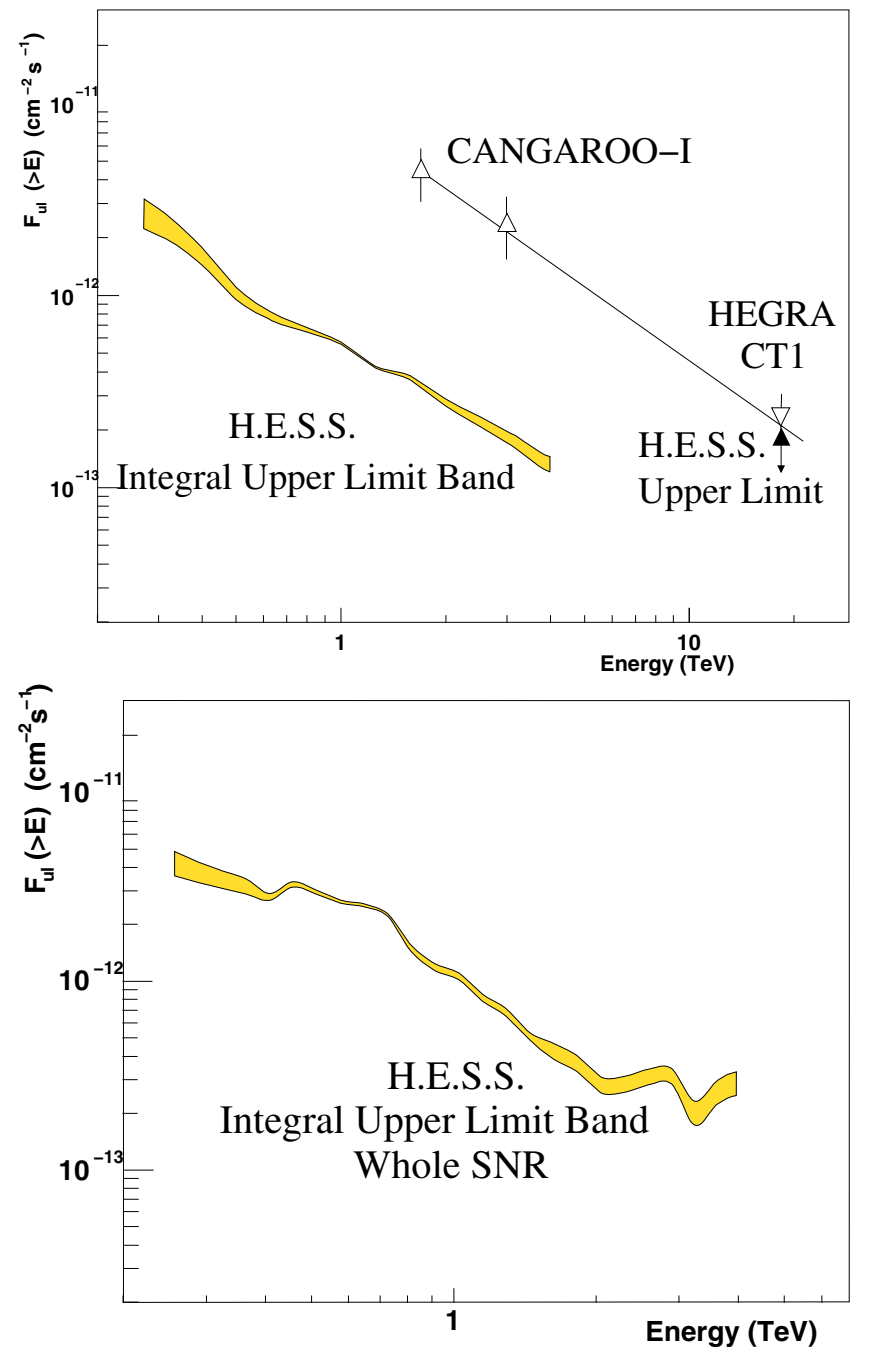

Fig. 2. Top: comparison of experimental results for SN1006 from HESS (integral upper limit $99.9 \%$ c.l. band at the CANGAROO position using $\theta_{\text {cut }}<0.23^{\circ}$, see Table 2), CANGAROO-I fluxes of Tanimori et al. (1998) and the HEGRA CT1 flux of Vitale et al. (2003). The solid line joins the two CANGAROO-I fluxes and is extended up to the HEGRA CT1 flux. The high energy HESS upper limit is shown for comparison with the HEGRA CT1 flux. The HESS UL band arises when assuming a differential spectral index from $\Gamma=2$ to 3 . Bottom: HESS upper limit band for the whole SNR, after cuts described in the text. The band arises when assuming a differential spectral index of $\Gamma=2$ to 3 .

4. CANGAROO Point 2004: as above using only 2004 data.

5. High Energy Point: excess events at the CANGAROO position using $\theta_{\text {cut }}<0.14^{\circ}$ cut appropriate to the HESS PSF but with an energy threshold $E>18 \mathrm{TeV}$ to compare with the HEGRA CT1 result (Vitale et al. 2003). Due to low statistics, the background is estimated from the full FoV (radius $2.0^{\circ}$ ) excluding the source region and corrected for differences in expected FoV efficiency. The dominant error comes from the on-count statistics.

6. Whole SNR: entire SNR at the remnant centre using $\theta_{\text {cut }}<$ $0.50^{\circ}$ and HESS energy threshold of $(E>0.26 \mathrm{TeV})$.

7. SW Rim: as for Position 1 but centered on the SE rim. 
Table 2. Event statistics and flux upper limits (using the method of Feldman \& Cousins 1998, assuming a differential photon index $\Gamma=2$ ) for a number of a priori-chosen regions associated with SN 1006: $s$-source events; $b$ - CR background estimate; $\alpha$ - Normalisation factor; See text for an explanation of the various regions. Except for Limits 3 and 4, numbers are derived exclusively from combined 2003 and 2004 HESS data.

\begin{tabular}{|c|c|c|c|c|c|c|c|c|}
\hline Region & $\mathrm{RA}^{a}$ & $\operatorname{Dec}^{a}$ & $\theta_{\text {cut }}$ & $s$ & $b$ & $\alpha$ & $\mathrm{S}^{b}$ & $\phi^{99.9 \% c}$ \\
\hline $\begin{array}{l}\text { 1. HESS Point }(E>0.26 \mathrm{TeV}) \\
\text { (also NE rim) }\end{array}$ & $15^{\mathrm{h}} 03^{\mathrm{m}} 48^{\mathrm{s}}$ & $-41^{\circ} 45^{\prime}$ & $0.14^{\circ}$ & 1072 & 6854 & 0.150 & 1.19 & 2.19 \\
\hline 2. CANGAROO Point $(E>1.7 \mathrm{TeV})$ & $15^{\mathrm{h}} 03^{\mathrm{m}} 48^{\mathrm{s}}$ & $-41^{\circ} 45^{\prime}$ & $0.25^{\circ}$ & 127 & 792 & 0.153 & 0.48 & 0.34 \\
\hline $\begin{array}{l}\text { 3. CANG. Point }(E>1.7 \mathrm{TeV}) \\
\text { (2003 HESS Data) }\end{array}$ & $15^{\mathrm{h}} 03^{\mathrm{m}} 48^{\mathrm{s}}$ & $-41^{\circ} 45^{\prime}$ & $0.25^{\circ}$ & 56 & 309 & 0.152 & 1.2 & 0.45 \\
\hline $\begin{array}{l}\text { 4. CANG. Point }(E>1.7 \mathrm{TeV}) \\
\text { (2004 HESS Data) }\end{array}$ & $15^{\mathrm{h}} 03^{\mathrm{m}} 48^{\mathrm{s}}$ & $-41^{\circ} 45^{\prime}$ & $0.25^{\circ}$ & 71 & 483 & 0.154 & -0.35 & 0.49 \\
\hline 5. H.E. Point $(E>18 \mathrm{TeV})$ & $15^{\mathrm{h}} 03^{\mathrm{m}} 48^{\mathrm{s}}$ & $-41^{\circ} 45^{\prime}$ & $0.25^{\circ}$ & 0 & 20 & 0.0069 & - & $0.18^{d}$ \\
\hline 6. Whole SNR $(E>0.26 \mathrm{TeV})$ & $15^{\mathrm{h}} 02^{\mathrm{m}} 48.4^{\mathrm{s}}$ & $-41^{\circ} 54^{\prime} 42^{\prime \prime}$ & $0.50^{\circ}$ & 13358 & 63113 & 0.217 & -2.8 & 2.39 \\
\hline 7. SW $\operatorname{Rim}(E>0.26 \mathrm{TeV})$ & $15^{\mathrm{h}} 02^{\mathrm{m}} 4^{\mathrm{s}}$ & $-42^{\circ} 06^{\prime} 3^{\prime \prime}$ & $0.14^{\circ}$ & 1042 & 6754 & 0.150 & 0.80 & 1.98 \\
\hline
\end{tabular}

${ }^{a}$ RA and Dec J2000.0 epoch.

${ }^{b}$ Significance from Eq. (17) of Li \& Ma (1983), using normalisation factor $\alpha$.

${ }^{c} \phi_{\mathrm{ph}}^{99.9 \%}=99.9 \%$ integral upper limit $\left(\times 10^{-12} \mathrm{ph} \mathrm{cm}^{-2} \mathrm{~s}^{-1}\right)$.

${ }^{d}$ Dominant error is from $s$. We quote here the $99.9 \%$ upper limit, which for a Poisson of mean zero is 7 counts.

\section{Discussion and conclusions}

Comparing the HESS upper limits with the fluxes from CANGAROO-I (1996 and 1997 observations) reveals a discrepancy of a factor $\sim 10$ (see Fig. 2). It is also clear that no $\gamma$-ray emission arises in only one of the years that HESS has observed, 2003 nor 2004 (see results in Table 2 for the CANGAROO position, 2003 and 2004 respectively). Thus HESS observations do not confirm the previously-published CANGAROO fluxes for SN1006. One would have to invoke a $\gamma$-ray flux variation over timescales less than 1 percent of the SNR age of an order of magnitude, in order to explain its nondetection by HESS. This is however quite unlikely to occur within the long-accepted diffusive shock acceleration framework for particle acceleration in SNR.

The HEGRA CT1 telescope observed SN1006 at very large zenith angles and obtained a preliminary flux of $F(E>$ $18 \mathrm{TeV})=2.5 \pm 0.5_{\text {stat }} \times 10^{-13} \mathrm{ph} \mathrm{cm}^{-2} \mathrm{~s}^{-1}$ (Vitale et al. 2003). We applied a matching high threshold cut to HESS data and obtained zero counts on-source, (with $s-\alpha b=-0.14$ excess events). The $99.9 \%$ upper limit on this is a Poisson distribution with a mean of 7 events; we take here the dominant error in the on-source counts $s$ and quote the Poisson error. In comparing with the HEGRA CT1 result one must also consider the systematic uncertainty on this flux, estimated to be $\geq 35 \%$ (Vitale et al. 2003). Given our exposure and considering both statistical and (uncorrelated) systematic uncertainties, the HEGRA CT1 flux would yield $11 \pm 6$ excess counts if seen by HESS. The lower fluctuated value, at 5 counts, is consistent with our observed zero counts with a chance probability of $\sim 7 \times 10^{-3}$. We also include the $99.9 \%$ upper limit in Fig. 2 for the whole SNR as a function of energy. This allows comparison in cases where emission from models is predicted from regions not necessarily restricted to the NE and SW rims.

The HESS upper limits permit estimation of important parameters for SN1006 in the framework of this source as a particle accelerator. One can first estimate an upper limit to the $\gamma$-ray luminosity as $L_{\gamma}=4 \pi F d^{2} \mathrm{erg} \mathrm{s}^{-1}$ for a $\gamma$-ray flux $F$ and source distance $d$. Distance estimates for SN1006 are in the range $d \sim$ 0.7 to $2.0 \mathrm{kpc}$ (see Allen et al. 2001 for a recent summary of measurements). Taking the HESS upper limit for the whole SNR $(E>0.26 \mathrm{TeV})$, distance $d=2.0 \mathrm{kpc}$ and assuming both a differential photon spectrum of $\Gamma=2$ and an energy range 0.26 to $10 \mathrm{TeV}$ gives $L_{\gamma}<1.7 \times 10^{33} \mathrm{erg} \mathrm{s}^{-1}$. This applies to the present epoch of SNR evolution for SN1006, which is likely in the early Sedov phase (Berezhko et al. 2002). We also estimate the energy in the corresponding accelerated protons $W_{\mathrm{p}}$ over the SNR lifetime as $W_{\mathrm{p}} \sim L_{\gamma} \tau_{\mathrm{pp}}$ erg. The $W_{\mathrm{p}}$ estimate applies to protons of energy $E_{\mathrm{p}} \sim 1.5$ to $60 \mathrm{TeV}$ and accounts for the average inelasticity $(\sim 0.17)$ of converting energy from protons to $\gamma$-rays in this scheme. The rather energy independent characteristic lifetime $\tau_{\mathrm{pp}}$ of accelerated protons interacting with an ambient matter of density $n \mathrm{~cm}^{-3}\left(\mathrm{p}+\mathrm{p} \rightarrow \pi^{0}+X \rightarrow 2 \gamma+X\right)$ is $\tau_{\mathrm{pp}} \sim 4.5 \times 10^{15}\left(\mathrm{n} / \mathrm{cm}^{-3}\right)^{-1} \mathrm{~s}$. With $L_{\gamma}$ derived above we arrive at $W_{\mathrm{p}}<7.8 \times 10^{48}\left(\mathrm{n} / \mathrm{cm}^{-3}\right)^{-1} \mathrm{erg}$. Values for $n$ are in the range $n=0.05$ to $\sim 0.3 \mathrm{~cm}^{-3}$ based on X-ray and optical observations. Adopting the lowest value $n=0.05 \mathrm{~cm}^{-3}$ of the range of densities yields the upper limit $W_{\mathrm{p}}<1.6 \times 10^{50} \mathrm{erg}$. The choice of $n$ in the $W_{\mathrm{p}}$ estimate should reflect the average density of ambient matter inside the remnant undergoing interaction with the downstream cosmic-rays, which are assumed to be confined inside the SNR. A lower limit on this value would be that of the ambient matter. We can account for the fact that the energy band of HESS observations corresponding 
to protons $(\sim 1.5$ to $60 \mathrm{TeV})$ is a fraction $\sim 0.25$ of the total expected energy in protons over a much wider range of energies covering the cosmic-ray spectrum up to the knee (we assume here $\sim 1 \mathrm{GeV}$ to $3 \mathrm{PeV}$ with a differential particle index of -2 ). Given this, an upper limit on the full proton budget would be $W_{\mathrm{p}}<6.3 \times 10^{50}$ erg.

Under the synchrotron/IC (scattering on the ubiquitous cosmic microwave background) scenario, knowledge of the synchrotron X-ray flux $f_{\mathrm{x}}$ and $\mathrm{TeV}$ IC flux $f_{\gamma}$ arising from the same electrons permits a clear constraint on the $B$ field in the shock-compressed regions (downstream from the shock) of the SNR according to $f_{\gamma}\left(E_{\gamma}\right) / f_{\mathrm{x}}\left(E_{\mathrm{x}}\right) \sim 0.1(B / 10 \mu \mathrm{G})^{-2} \xi$ (Aharonian et al. 1997). It is assumed here that the X-ray and $\gamma$-ray emitting regions are the same size and thus that the filling factor $\xi \sim 1$. Such a case arises for high $B$ fields when electrons rapidly cool at their synchrotron production regions. High values for $B>40 \mu \mathrm{G}$ are in fact implied by X-ray observations and by subsequent interpretation in the diffusive shock acceleration framework (Allen et al. 2001; Berezhko et al. 2002, 2003; Bamba et al. 2003; Yamazaki et al. 2004; Ksenofontov et al. 2004). With the HESS upper limits on the $\gamma$-ray emission, one can therefore estimate a lower limit on the $B$-field. The synchrotron $\left(E_{\mathrm{x}}\right)$ and IC $\left(E_{\gamma}\right)$ photon energies are coupled according to $E_{\gamma} \sim 1.5\left(E_{\mathrm{x}} / 0.1 \mathrm{keV}\right)(B / 10 \mu \mathrm{G})^{-1} \mathrm{TeV}$, which requires that fluxes $f_{\mathrm{x}}$ and $f_{\gamma}$ in appropriate energy ranges be compared. Comparing the available X-ray energy flux ( $E=0.1$ to $2 \mathrm{keV}$; Allen et al. 2001) $f_{\mathrm{x}}=1.42 \times 10^{-10} \mathrm{erg} \mathrm{cm}^{-2} \mathrm{~s}^{-1}$ with that of HESS at $f_{\gamma}=2.29 \times 10^{-12} \mathrm{erg} \mathrm{cm}^{-2} \mathrm{~s}^{-1}$ (for the whole SNR over the energy range $E \sim 1$ to $10 \mathrm{TeV}$ and assuming a -2.0 spectral index) yields a lower limit of $B>25 \mu \mathrm{G}$. This lower limit on $B$ is consistent with values discussed earlier, which result from comparisons of X-ray observations with detailed theory. In summary, HESS observations of SN1006 have not revealed evidence for $\mathrm{TeV} \gamma$-ray emission, and the resulting upper limits will be valuable in further constraining the parameters of $\gamma$-ray production in SN1006 (see e.g. Aharonian \& Atoyan 1999; Berezhko et al. 2002; Ksenofontov et al. 2004).

Acknowledgements. The support of the Namibian authorities and of the University of Namibia in facilitating the construction and operation of HESS is gratefully acknowledged, as is the support by the German Ministry for Education and Research (BMBF), the Max Planck Society, the French Ministry for Research, the CNRS-IN2P3 and the Astroparticle Interdisciplinary Programme of the CNRS, the UK Particle Physics and Astronomy Research Council (PPARC), the IPNP of the Charles University, the South African Department of Science and Technology and National Research Foundation, and by the University of Namibia. We appreciate the excellent work of the technical support staff in Berlin, Durham, Hamburg, Heidelberg, Palaiseau, Paris, Saclay, and in Namibia in the construction and operation of the equipment.

\section{References}

Aharonian, F. A., \& Atoyan, A. M. 1999, A\&A, 351, 330

Aharonian, F. A., Atoyan, A. M., \& Kifune, T. 1997, MNRAS, 291, 162

Aharonian, F. A., Akhperjanian, A. J., Aye, K. M., et al. 2005, A\&A, in preparation

Allen, G. E., Petre, R., \& Gotthelf, E. V. 2001, ApJ, 558, 739

Bamba, A., Ueno, M., Nakajima, H., \& Koyama, K. 2003, ApJ, 589, 827

Berezhko, E. G., Ksenofontov, L. T., \& Völk, H. J. 2002, A\&A, 395, 943

Berezhko, E. G., Ksenofontov, L. T., \& Völk, H. J. 2003, A\&A, 412, L11

Bernlöhr, K., Carrol, O., Cornils, R., et al. 2003, Astropart. Phys., 20, 111

Cornils, R., Gillessen, S., Jung, I., et al. 2003, Astropart. Phys., 20, 129

Drury, L. O'C., Aharonian, F. A., \& Völk, H. J. 1994, A\&A, 287, 959

Feldman, G. J., \& Cousins, R. D. 1998, Phys. Rev., D57, 3837

Funk, S., Hermann, G., Hinton, J., et al. 2004, Astropart. Phys., 22, 285

Hara, S., Tanimori, T., Kubo, H., et al. 2001, in Proc. 27th ICRC Hamburg, 5, 2455

Hillas, A. 1985, in Proc. 19th ICRC (La Jolla), 3, 445

Hofmann, W., Jung, I., Konopelko, A., et al. 1999, Astropart. Phys., 10,275

Koyama, K., Petre, R., Gotthelf, E. V., et al. 1995, Nature, 378

Ksenofontov, L. T., Berezhko, E. G., \& Völk, H. J. 2004, in Proc. Symp. on High Energy Gamma-Ray Astronomy (Heidelberg, AIP Press), in press

Li, T. P., \& Ma, Y. Q. 1983, ApJ, 272, 317

Long, K. S., Reynolds, S. P., Raymond, J. C., et al. 2003, ApJ, 586, 1162

Naito, T., \& Takahara, F. 1994, J. Phys. G: Nucl. Part. Phys., 20, 477

Naito, T., Yoshida, T., Mori, M., \& Tanimori, T. 1999, Astron. Nachr., 320,205

Masterson, C., et al. 2003, Proc. 28th ICRC Tsukuba OG2.2, 2323

Rowell, G. P. 2003, A\&A, 410, 389

Tanimori, T., Hayami, Y., Kamei, S., et al. 1998, ApJ, L25

Tanimori, T., Naito, T., Yoshida, T., et al. 2001, in Proc. 27th ICRC Hamburg, 5, 2465

Vincent, P., et al. 2003, Proc. 28th ICRC, Tsukuba (Univ. Academy Press Tokyo), 2887

Vitale, V., et al. 2003, in Proc. 28th ICRC Tsukuba, OG2.2, 2389

Yamazaki, R., Yoshida, T., Terasawa, T., et al. 2004, A\&A, 416, 595 